\title{
Exploring concordance and discordance for return of incidental findings from clinical sequencing
}

\author{
Robert C. Green, MD, MPH ${ }^{1,2}$, Jonathan S. Berg, MD, PhD³, Gerard T. Berry, MD ${ }^{4,5}$, \\ Leslie G. Biesecker, $\mathrm{MD}^{6}$, David P. Dimmock, MD', James P. Evans, MD, PhD ${ }^{3}$, \\ Wayne W. Grody, MD, PhD ${ }^{8-10}$, Madhuri R. Hegde, PhD ${ }^{11}$, Sarah Kalia, ScM1, \\ Bruce R. Korf, MD, PhD ${ }^{12}$, Ian Krantz, MD ${ }^{13}$, Amy L. McGuire, JD, PhD ${ }^{14}$, \\ David T. Miller, MD, PhD ${ }^{4,15}$, Michael F. Murray, MD ${ }^{1,2}$, Robert L. Nussbaum, MD ${ }^{16}$, \\ Sharon E. Plon, MD, PhD ${ }^{17,18}$, Heidi L. Rehm, PhD ${ }^{2,19}$ and Howard J. Jacob, $\mathrm{PhD}^{7,20}$
}

Purpose: The aim of this study was to explore specific conditions and types of genetic variants that specialists in genetics recommend should be returned as incidental findings in clinical sequencing.

Methods: Sixteen specialists in clinical genetics and/or molecular medicine selected variants in 99 common conditions to return to the ordering physician if discovered incidentally through whole-genome sequencing. For most conditions, the specialists independently considered three molecular scenarios for both adults and minor children: a known pathogenic mutation, a truncating variant presumed pathogenic (where other truncating variants are known to be pathogenic), and a missense variant predicted in silico to be pathogenic.

Results: On average, for adults and children, respectively, each specialist selected 83.5 and 79.0 conditions or genes of 99 in the known pathogenic mutation categories, 57.0 and 53.5 of 72 in the truncating variant categories, and 33.4 and 29.7 of 72 in the missense variant categories. Concordance in favor of disclosure within the adult/ known pathogenic mutation category was $100 \%$ for 21 conditions or genes and $80 \%$ or higher for 64 conditions or genes.

Conclusion: Specialists were highly concordant for the return of findings for 64 conditions or genes if discovered incidentally during whole-exome sequencing or whole-genome sequencing.

Genet Med 2012:14(4):405-410

Key Words: incidental findings; whole-exome sequencing; wholegenome sequencing

out, interpret, and communicate incidental or secondary (i.e., unrelated to reasons for ordering) genetic findings.

Whatever the clinical indication for WES/WGS, there is considerable potential in each patient to discover large numbers of variants in genes associated with human disease. ${ }^{16}$ Currently, there are no guidelines for return of incidental findings from clinical sequencing, although there have been proposals for lower- and higher-risk categories or "bins" based on clinical validity and actionability. ${ }^{17}$ Yet no one has asked whether wellmeaning specialists would agree upon incidental findings that would be appropriate to disclose. To explore concordance or discordance that such efforts might face, 16 specialists in genetics independently evaluated 99 common genetic conditions and

\footnotetext{
${ }^{1}$ Division of Genetics, Department of Medicine, Brigham and Women's Hospital and Harvard Medical School, Boston, Massachusetts, USA; ${ }^{2}$ Partners Center for Personalized Genetic Medicine, Boston, Massachusetts, USA; ${ }^{3}$ Department of Genetics, University of North Carolina at Chapel Hill School of Medicine, Chapel Hill, North Carolina, USA; ${ }^{4}$ Division of Genetics, Children's Hospital Boston and Harvard Medical School, Boston, Massachusetts, USA; ${ }^{5}$ The Manton Center for Orphan Disease Research, Children's Hospital Boston, Boston, Massachusetts, USA; ${ }^{6}$ National Human Genome Research Institute, National Institutes of Health, Bethesda, Maryland, USA; ${ }^{7}$ Division of Genetics, Department of Pediatrics, Medical College of Wisconsin, Milwaukee, Wisconsin, USA; ${ }^{8}$ Division of Medical Genetics, Department of Human Genetics, UCLA School of Medicine, Los Angeles, California, USA; ${ }^{9}$ Division of Molecular Pathology, Department of Pathology \& Laboratory Medicine, UCLA School of Medicine, Los Angeles, California, USA; ${ }^{10}$ Division of Pediatric Genetics, Department of Pediatrics, UCLA School of Medicine, Los Angeles, California, USA; ${ }^{11}$ Department of Human Genetics, Emory University School of Medicine, Atlanta, Georgia, USA; ${ }^{12}$ Department of Genetics, University of Alabama, Birmingham, Alabama, USA; ${ }^{13}$ Division of Human Genetics and Molecular Biology, The Children's Hospital of Philadelphia, Philadelphia, Pennsylvania, USA; ${ }^{14}$ Center for Medical Ethics and Health Policy, Baylor College of Medicine, Houston, Texas, USA; ${ }^{15}$ Department of Laboratory Medicine, Children's Hospital Boston and Harvard Medical School, Boston, Massachusetts, USA; ${ }^{16}$ Division of Medical Genetics, Department of Medicine, and Institute for Human Genetics, University of California, San Francisco, San Francisco, California, USA; ${ }^{17}$ Department of Pediatrics, Baylor College of Medicine, Houston, Texas, USA; ${ }^{18}$ Department of Molecular and Human Genetics, Baylor College of Medicine, Houston, Texas, USA; ${ }^{19}$ Department of Pathology, Brigham and Women's Hospital and Harvard Medical School, Boston, Massachusetts, USA; ${ }^{20}$ Department of Physiology, Medical College of Wisconsin, Milwaukee, Wisconsin, USA. Correspondence: Robert C. Green (rcgreen@genetics.med.harvard.edu)
} 
individual genes and selected those they would recommend reporting back to the patient's physician as incidental findings after WGS.

\section{MATERIALS AND METHODS}

A list was generated of all diseases for which testing is clinically available as registered on the GeneTests website..$^{18}$ Genes associated with the same disease were combined. The top 88 conditions or genes based on the frequency of laboratory testing were supplemented by adding hereditary breast and ovarian cancer, a condition that was not frequent among laboratories due to patented genes, along with a number of common chromosomal conditions and deletion syndromes currently diagnosed through cytogenetic analysis (Table $\mathbf{1}$ ).

The 16 participating specialists were clinical geneticists and/ or molecular laboratory directors, most of whom have been involved in early uses of genome-scale data, but there was no attempt to be representative or to include all relevant subspecialists. Each specialist was asked to assume that he/she was serving as a consultant to a laboratory that performs clinical WGS and to decide which variants discovered as incidental findings should be returned in a report to the ordering physician. Specialists were asked to assume that family history was not available, that the patient had no previously recognized clinical features consistent with the disease variant under consideration, that the patient's gender was known, and that it was known whether the patient was an adult or a minor child (under 18 years), but not the exact age. Specialists were asked to assume that the sequencing was perfectly accurate and could detect translocations and repeat expansions perfectly, even though this degree of accuracy is not available through current WES/WGS technologies. Details of the consent process, patient preferences regarding results disclosure, and details about how disclosure of results might be handled by the referring physician were not specified. An additional assumption was that family members of the patient being tested would not have been sequenced or genetically tested for the variant under consideration, thus decisions were made on the basis of the genome findings alone without contextualization by patient medical history or family history.

Specialists provided separate responses as to whether incidental findings should be returned to the physicians of adults and of minor children, and where applicable, in each of three categories of variants: a known pathogenic mutation, a truncating variant presumed pathogenic (where other truncating variants were known to be pathogenic), and a missense variant predicted to be deleterious by in silico analysis. For recessive conditions or genes, specialists were asked to specify whether they would return a variant even in a carrier state, or only if the patient were found to be biallelic (for autosomal recessive) or hemizygous (for X-linked), or not at all. For repeat-expansion disorders, specialists indicated whether they would return a finding of a premutation or full mutation, only a full mutation, or neither. For each possible scenario per disease (adult/child, pathogenic/truncating/missense), specialists were also asked if they had difficulty deciding whether to report the variant. Each specialist could decline to respond due to lack of familiarity with the condition or gene. All of the aforementioned choices were made through pull-down, forced-choice menus. For the purposes of this analysis, conditions or genes were counted if the specialist recommended an affirmative response in the dominant disorders or genes, or in any option of the recessive, $\mathrm{X}$-linked, or expansion disorders or genes.

The specialists did not communicate with each other about their decisions. All 16 specialists are listed as coauthors of this paper. One additional coauthor (S.K.) is a genetic counselor who prepared the lists and assisted with data analyses and manuscript preparation, and a second additional coauthor (A.L.M.) is a contributor in ethics and legal issues who participated in data analyses and manuscript preparation.

\section{RESULTS}

Of the 16 specialists who selected conditions, 13 were MDs with or without an additional degree, and 3 were non-MD PhDs; 9 were primarily clinical geneticists, 3 were primarily molecular laboratorians, and 4 were active both clinically and in the molecular laboratory.

The conditions and genes proposed and the number and percentage of specialists who would recommend return of incidental findings based on these are shown in Table 1 (specialist order does not correlate with order of coauthors). For each of the 99 conditions and genes, an average of 13.5 (s.d. 1.9, range 8-16) specialists suggested incidental genetic findings be returned about adults and 12.8 (s.d. 2.3, range 4-16) suggested findings be returned about children if there was a known pathogenic mutation. For each of 72 conditions and genes (excluding trinucleotide repeat-expansion, chromosomal, and deletion conditions, and conditions caused by only a specific known mutation), an average of 12.6 specialists (s.d. 2.3, range 8-16) and 11.9 specialists (s.d. 2.2, range 7-15) suggested that incidental findings of truncating variants be returned about adults and children, respectively. An average of 7.4 specialists (s.d. 1.5, range 4-10) and 6.6 specialists (s.d. 1.7, range 3-9) suggested that incidental findings of a missense variant predicted to be pathogenic by in silico analysis be returned about adults and children, respectively. The concordance in favor of disclosure was 100\% for 21 conditions or genes in the Adult/Known Pathogenic Mutation category, 80\% or higher for 64 conditions or genes, and at least $50 \%$ for all conditions or genes.

Concordance was higher for returning incidental information about conditions that had potential for medical intervention (such as cancer predisposition syndromes) than for those without such potential (such as developmental or neurodegenerative disorders). Concordance was also higher when returning incidental findings about adults rather than about children, and for returning known pathogenic mutations and presumed pathogenic truncating variants rather than missense variants predicted to be pathogenic. The total number of genes or conditions selected by each specialist varied along these 
Table 1 Number (\%) of specialists selecting each incidental genetic finding for return

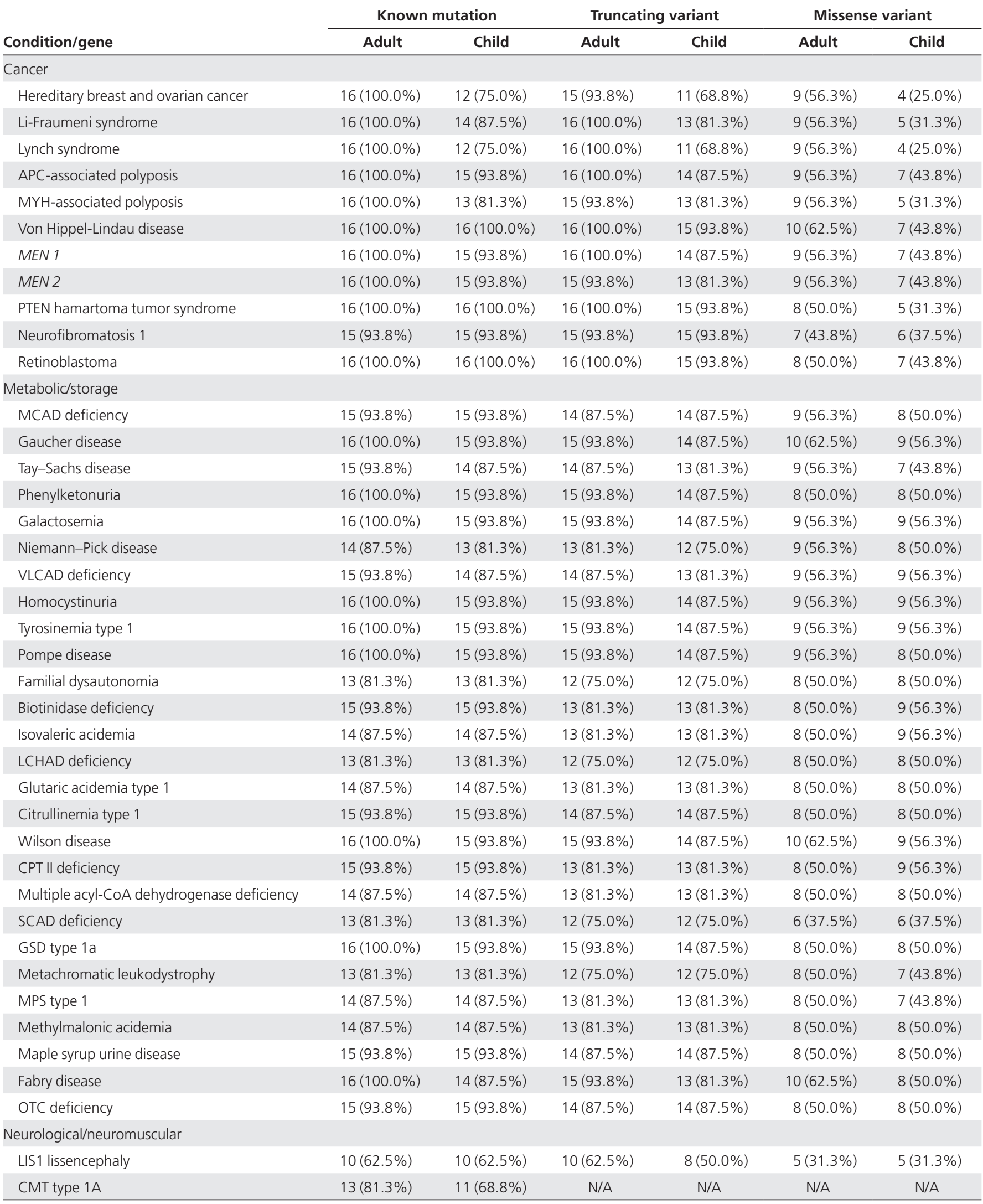

N/A, not applicable. 
Table 1 Continued

\begin{tabular}{|c|c|c|c|c|c|c|}
\hline \multirow[b]{2}{*}{ Condition/gene } & \multicolumn{2}{|c|}{ Known mutation } & \multicolumn{2}{|c|}{ Truncating variant } & \multicolumn{2}{|c|}{ Missense variant } \\
\hline & Adult & Child & Adult & Child & Adult & Child \\
\hline CMT type 1B & $13(81.3 \%)$ & $11(68.8 \%)$ & $10(62.5 \%)$ & $9(56.3 \%)$ & $5(31.3 \%)$ & $3(18.8 \%)$ \\
\hline Early onset primary dystonia & $11(68.8 \%)$ & $10(62.5 \%)$ & $8(50.0 \%)$ & $7(43.8 \%)$ & $5(31.3 \%)$ & $5(31.3 \%)$ \\
\hline Epileptic encephalopathy infantile 2 & $9(56.3 \%)$ & $9(56.3 \%)$ & $8(50.0 \%)$ & $7(43.8 \%)$ & $5(31.3 \%)$ & $5(31.3 \%)$ \\
\hline Dystrophinopathies & $14(87.5 \%)$ & $13(81.3 \%)$ & $12(75.0 \%)$ & $11(68.8 \%)$ & $6(37.5 \%)$ & $5(31.3 \%)$ \\
\hline CMT type $\mathrm{X} 1$ & $12(75.0 \%)$ & $11(68.8 \%)$ & $10(62.5 \%)$ & $9(56.3 \%)$ & $5(31.3 \%)$ & $3(18.8 \%)$ \\
\hline ARX-related & $11(68.8 \%)$ & $10(62.5 \%)$ & N/A & $\mathrm{N} / \mathrm{A}$ & $\mathrm{N} / \mathrm{A}$ & N/A \\
\hline Huntington disease & $10(62.5 \%)$ & $5(31.3 \%)$ & N/A & N/A & N/A & N/A \\
\hline Myotonic dystrophy type 1 & $14(87.5 \%)$ & $13(81.3 \%)$ & N/A & N/A & N/A & N/A \\
\hline Friedreich ataxia & $12(75.0 \%)$ & $13(81.3 \%)$ & N/A & $\mathrm{N} / \mathrm{A}$ & N/A & N/A \\
\hline \multicolumn{7}{|l|}{ Mitochondrial } \\
\hline Leber hereditary optic neuropathy & $12(75.0 \%)$ & $11(68.8 \%)$ & $10(62.5 \%)$ & $9(56.3 \%)$ & $6(37.5 \%)$ & $5(31.3 \%)$ \\
\hline MELAS & $12(75.0 \%)$ & $12(75.0 \%)$ & $9(56.3 \%)$ & $9(56.3 \%)$ & $4(25.0 \%)$ & $4(25.0 \%)$ \\
\hline MERRF & $12(75.0 \%)$ & $12(75.0 \%)$ & $9(56.3 \%)$ & $9(56.3 \%)$ & $4(25.0 \%)$ & $4(25.0 \%)$ \\
\hline Leigh syndrome and NARP & $12(75.0 \%)$ & $12(75.0 \%)$ & $10(62.5 \%)$ & $10(62.5 \%)$ & $5(31.3 \%)$ & $5(31.3 \%)$ \\
\hline \multicolumn{7}{|l|}{ Developmental } \\
\hline Noonan syndrome & $12(75.0 \%)$ & $12(75.0 \%)$ & $11(68.8 \%)$ & $11(68.8 \%)$ & $5(31.3 \%)$ & $5(31.3 \%)$ \\
\hline \multicolumn{7}{|l|}{ Inherited predisposition (noncancer) } \\
\hline Factor V Leiden thrombophilia & $12(75.0 \%)$ & $9(56.3 \%)$ & N/A & $\mathrm{N} / \mathrm{A}$ & $\mathrm{N} / \mathrm{A}$ & N/A \\
\hline Prothrombin-related thrombophilia & $11(68.8 \%)$ & $9(56.3 \%)$ & $\mathrm{N} / \mathrm{A}$ & $\mathrm{N} / \mathrm{A}$ & $\mathrm{N} / \mathrm{A}$ & N/A \\
\hline Familial hypercholesterolemia & $16(100.0 \%)$ & $14(87.5 \%)$ & $13(81.3 \%)$ & $11(68.8 \%)$ & $7(43.8 \%)$ & $6(37.5 \%)$ \\
\hline Hereditary hemochromatosis & $14(87.5 \%)$ & $10(62.5 \%)$ & N/A & N/A & N/A & N/A \\
\hline MTHFR & $9(56.3 \%)$ & $8(50.0 \%)$ & N/A & N/A & N/A & N/A \\
\hline a1-Antitrypsin deficiency & $15(93.8 \%)$ & $13(81.3 \%)$ & N/A & $\mathrm{N} / \mathrm{A}$ & N/A & N/A \\
\hline Gilbert syndrome & $11(68.8 \%)$ & $9(56.3 \%)$ & $10(62.5 \%)$ & $8(50.0 \%)$ & $5(31.3 \%)$ & $4(25.0 \%)$ \\
\hline$A P O E$ & $8(50.0 \%)$ & $4(25.0 \%)$ & N/A & N/A & N/A & N/A \\
\hline \multicolumn{7}{|l|}{ Other } \\
\hline Achondroplasia & $12(75.0 \%)$ & $12(75.0 \%)$ & $\mathrm{N} / \mathrm{A}$ & N/A & $\mathrm{N} / \mathrm{A}$ & N/A \\
\hline Hypochondroplasia & $12(75.0 \%)$ & $13(81.3 \%)$ & N/A & $\mathrm{N} / \mathrm{A}$ & $\mathrm{N} / \mathrm{A}$ & N/A \\
\hline Marfan syndrome & $15(93.8 \%)$ & $15(93.8 \%)$ & $13(81.3 \%)$ & $13(81.3 \%)$ & $7(43.8 \%)$ & $7(43.8 \%)$ \\
\hline Dilated cardiomyopathy & $14(87.5 \%)$ & $13(81.3 \%)$ & $12(75.0 \%)$ & $12(75.0 \%)$ & $7(43.8 \%)$ & $7(43.8 \%)$ \\
\hline Romano-Ward (long QT syndrome) & $16(100.0 \%)$ & $16(100.0 \%)$ & $14(87.5 \%)$ & $14(87.5 \%)$ & $7(43.8 \%)$ & $7(43.8 \%)$ \\
\hline DFNA 3 nonsyndromic hearing loss & $12(75.0 \%)$ & $10(62.5 \%)$ & $9(56.3 \%)$ & $7(43.8 \%)$ & $7(43.8 \%)$ & $7(43.8 \%)$ \\
\hline
\end{tabular}

N/A, not applicable. 
Table 1 Continued

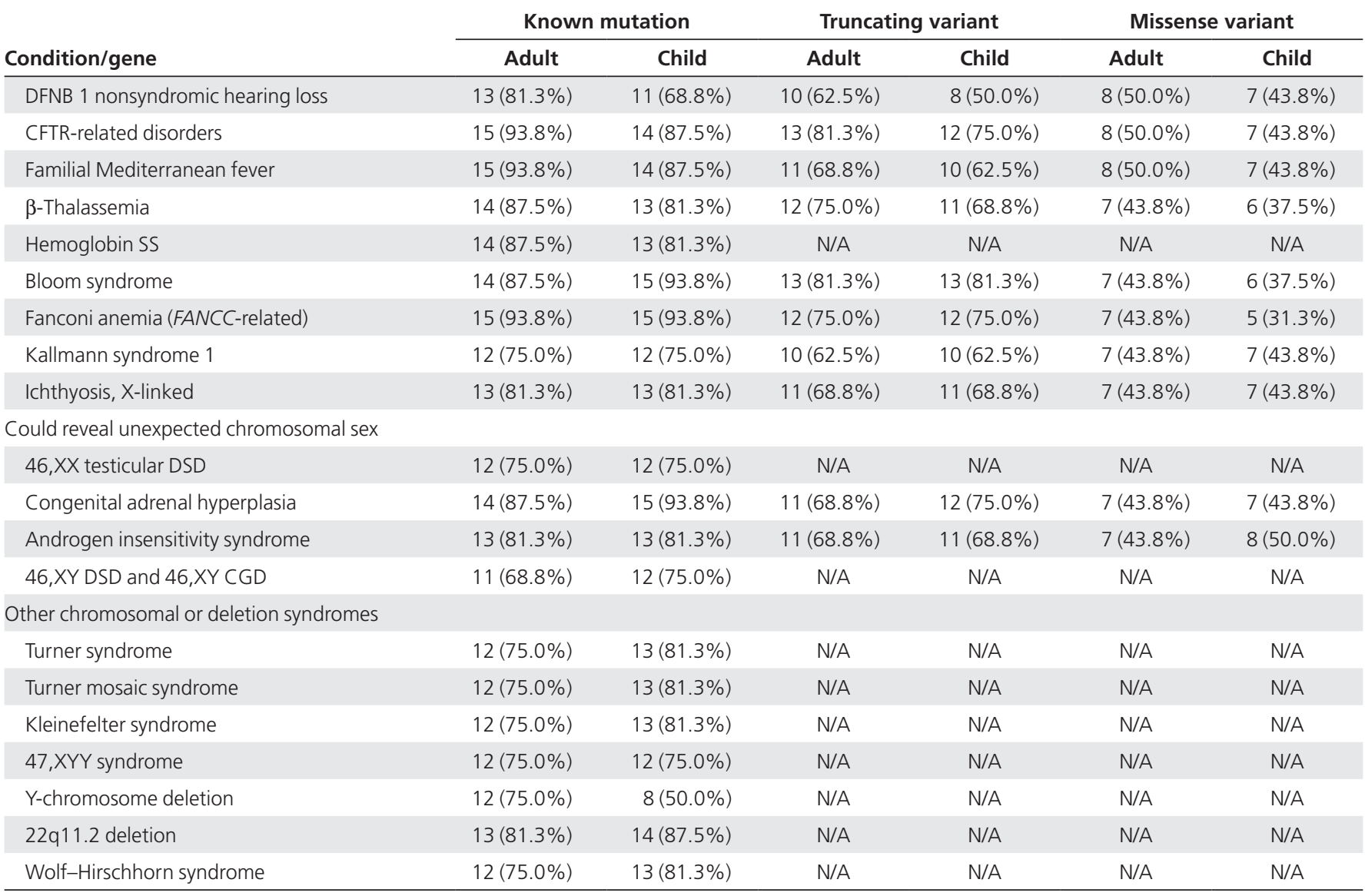

N/A, not applicable.

axes (Supplementary Table S1 online and Supplementary Figure S1 online) whereas the discomfort that specialists felt in making the decision varied inversely along the same axes (Supplementary Table S2 online).

\section{DISCUSSION}

This report presents an exploratory description of concordance and discordance around specific conditions and genes with respect to incidental findings in the context of clinical WGS/ WES. There were 21 conditions or genes in which all 16 specialists agreed that known pathogenic mutations should be disclosed if found incidentally in adults, and 4 conditions for which all 16 specialists agreed that known pathogenic mutations should be disclosed if found incidentally in minor children (Supplementary Table S3 online). There was considerable concordance among all 99 conditions or genes for known pathogenic mutations, with a majority of specialists selecting nearly every condition or gene for return as an incidental finding.

Substantial discordance was observed across two major domains. First, some specialists were more reluctant than others to disclose incidental findings. For example, even within the category of adult/known pathogenic mutations, one specialist reported that he or she would recommend disclosure of only $30.3 \%$ of the identified genes or conditions, whereas two others thought $100 \%$ should be disclosed. Second, there was discordance in judgments about the relative value of different criteria when making decisions about disclosures. For some, whether the result pertained to a child or an adult seemed to be the most relevant factor driving the decision to disclose, whereas for others, the established pathogenicity of the variant was the most important factor. These criteria also influenced whether contributors found a particular decision to be difficult. Additional research is needed to better understand the source of this discordance; for example, whether it reflects primarily differences in clinical or ethical judgment or, in the case of the potential pathogenicity of variants, whether specialists were fully aware of the limited accuracy of in silico prediction tools for missense variants.

These data have a number of limitations. The specialists were not representative and were of varying degrees of expertise. Each specialist responded independently and without interaction that would characterize formal consensus building. Specialists were asked to assume that no information was available about family history or knowledge of signs or symptoms in relatives of the proband, which is a somewhat artificial situation.

The degree of discordance observed suggests that whether considering incidental findings in research subjects or incidental findings in clinical WES/WGS, it may be difficult to reach 
consensus on a specific list of variants that meet a threshold for disclosure, as called for in various consensus statements on incidental findings. However, in an actual consensus group, experts on a subset of disorders would educate the rest of the group and likely increase concordance. Plans that attempt to factor in subject or patient preferences (which were excluded in this exercise) could make consensus easier or more difficult. In this exercise, the choice was whether to provide such information in a report to the ordering physician, not to the patient. This may have provided comfort to the specialists who were wavering because the ordering physician would have the ability to seek preferences from the patient about how much information to share, as well as the ability to contextualize the report by integrating personal medical history, family history, symptoms and signs, or by referring the patient. The variability in responses surrounding issues for which consensus has already been achieved and published (such as the unreliability of in silico prediction of variant significance) also highlights the need for greater education, even among specialists, as well as the need for extensively curated clinical-grade databases.

In summary, there was considerable concordance and considerable discordance in choices about return of incidental genomic information among specialists queried for this report. The variability in responses suggests that a relatively small panel of specialists is unlikely to embody all of the necessary expertise to achieve consensus about the vast number of genetic variants that will be identified by WES/WGS, especially if additional variants are considered that are less well characterized than those we selected. Modifications to a typical consensus approach may need to be considered, such as collaboration among multiple expert panels, with each panel being composed of specialists in a more narrow focus of expertise. It appears that even genetic specialists are a heterogeneous group and may not be yet fully prepared to deal with the implementation of new genetic technologies.

\section{SUPPLEMENTARY MATERIAL}

Supplementary material is linked to the online version of the paper at http://www.nature.com/gim

\section{ACKNOWLEDGMENT}

Funding for this work was provided by National Institutes of Health grants HG02213, AG027841, HG005491, HG005092, HG006615, HG003178, HG006500, CA138836, and HG006485. G.T.B. is supported by the Manton Center for Orphan Disease Research. L.G.B. is supported by intramural funding from the
National Human Genome Research Institute. A.L.M. is supported by the Baylor College of Medicine Clinical and Translational Research Program and the Baylor Annual Fund.

\section{DISCLOSURE}

The authors declare no conflict of interest.

\section{REFERENCES}

1. Guttmacher AE, McGuire AL, Ponder B, Stefánsson K. Personalized genomic information: preparing for the future of genetic medicine. Nat Rev Genet 2010;11:161-165.

2. Feero WG, Guttmacher AE, Collins FS. Genomic medicine-an updated primer. N Eng/ J Med 2010;362:2001-2011.

3. Ginsburg GS, Willard HF. Genomic and personalized medicine: foundations and applications. Trans/ Res 2009;154:277-287.

4. Korf BR. Genetics and genomics education: the next generation. Genet Med 2011;13:201-202.

5. Khoury MJ. Public health genomics: the end of the beginning. Genet Med 2011;13:206-209.

6. Green ED, Guyer MS. Charting a course for genomic medicine from base pairs to bedside. Nature 2011;470:204-213.

7. Mayer AN, Dimmock DP, Arca MJ, et al. A timely arrival for genomic medicine. Genet Med 2011;13:195-196.

8. Lupski JR, Reid JG, Gonzaga-Jauregui C, et al. Whole-genome sequencing in a patient with Charcot-Marie-Tooth neuropathy. N Engl J Med 2010;362:1181-1191.

9. Worthey EA, Mayer AN, Syverson GD, et al. Making a definitive diagnosis: successful clinical application of whole exome sequencing in a child with intractable inflammatory bowel disease. Genet Med 2011;13:255-262.

10. Link DC, Schuettpelz LG, Shen D, et al. Identification of a novel TP53 cancer susceptibility mutation through whole-genome sequencing of a patient with therapy-related AML. JAMA 2011;305:1568-1576.

11. Bainbridge MN, Wiszniewski W, Murdock DR, et al. Whole-genome sequencing for optimized patient management. Sci Trans/ Med 2011;3:87re3.

12. Berg JS, Evans JP, Leigh MW, et al. Next generation massively parallel sequencing of targeted exomes to identify genetic mutations in primary ciliary dyskinesia: implications for application to clinical testing. Genet Med 2011;13:218-229.

13. Baylor College of Medicine (BCM). Whole Genome Laboratory. http://www. bcm.edu/geneticlabs/index.cfm?PMID=21319. Accessed 10 November 2011.

14. Genome Web. Knome Adds CLIA-Certified SeqWright as Sequencing Provider for Personal Genome Services. http://www.genomeweb.com/ sequencing/knome-adds-clia-certified-seqwright-sequencing-providerpersonal-genome-services. Accessed 10 November 2011.

15. Genome Web. Partner's Healthcare Center's LMM to Introduce Clinical Whole-Genome Sequencing Interpretation Service in 2012. http://www. genomeweb.com/sequencing/partners-healthcare-centers-Imm-introduceclinical-whole-genome-sequencing-inter. Accessed 10 November 2011.

16. Kohane IS, Masys DR, Altman RB. The incidentalome: a threat to genomic medicine. JAMA 2006;296:212-215

17. Berg JS, Khoury MJ, Evans JP. Deploying whole genome sequencing in clinical practice and public health: meeting the challenge one bin at a time. Genet Med 2011;13:499-504.

18. GeneTests. Medical Genetics Information Resource, 2011. http://www. genetests.org. 